\title{
An Electronic Best Practice Alert Based on Choosing Wisely Guidelines Reduces Thrombophilia Testing in the Outpatient Setting
}

\author{
Tomi Jun, $M D^{7}$, Henry Kwang, $M D^{7}$, Eric Mou, $M D^{2}$, Caroline Berube, $M D^{2}$, Jason Bentley, $P h D^{3}$, \\ Lisa Shieh, $\mathrm{MD}^{4}$, and Jason Hom, MD
}

'Department of Medicine, Stanford University School of Medicine, Stanford, CA, USA; ${ }^{2}$ Department of Medicine, Division of Hematology, Stanford University School of Medicine, Stanford, CA, USA; ${ }^{3}$ Department of Medicine, Quantitative Sciences Unit, Stanford University School of Medicine, Stanford, CA, USA; ${ }^{4}$ Department of Medicine, Division of Hospital Medicine, Stanford University School of Medicine, Stanford, CA, USA.

KEY WORDS: thrombophilia; best practice alert; venous thromboembolism.

J Gen Intern Med 34(1):29-30

DOI: $10.1007 / \mathrm{s} 11606-018-4663-8$

(c) Society of General Internal Medicine 2018

\section{INTRODUCTION}

Thrombophilia testing in the setting of acute venous thromboembolism (VTE), including deep venous thrombosis (DVT) and pulmonary embolism (PE), is often inappropriate. Interpretation of many of the biochemical thrombophilia assays is confounded by anticoagulation or acute thrombus, and thrombophilia testing rarely affects immediate clinical decision-making. ${ }^{1-3}$

In an effort to curtail inappropriate thrombophilia testing, the American Society of Hematology (ASH) has issued Choosing Wisely guidelines recommending against thrombophilia testing in patients with VTE in the setting of major transient risk factors (surgery, trauma, and immobility). ${ }^{4}$ We evaluated the effect of a "Choosing Wisely" best practice alert (BPA) embedded into the electronic medical record (EMR) on thrombophilia testing patterns.

\section{METHODS}

This before-and-after study involved patients with a diagnosis of VTE seen at our center between January 1, 2016 and April 30, 2017. The study was approved by the Stanford University institutional review board.

The EMR-embedded BPA was activated in the outpatient setting on July 1, 2016 and in the inpatient setting on September 1,2016 . The BPA was triggered whenever any of nine prespecified thrombophilia tests were ordered for a non-pregnant adult patient with an encounter listing any of 120 pre-specified ICD-9 and ICD-10 codes related to acute or chronic VTE.

When triggered, the BPA appeared onscreen citing the ASH Choosing Wisely recommendation. Providers had the option of following or overriding the BPA. We tracked alert outcomes and compared adherence by setting, provider type, and provider specialty using Fisher's exact test.

Published online September 13, 2018
The thrombophilia testing rates (number of thrombophilia tests divided by number of VTE encounters per month) in the pre- and post-BPA periods were calculated using billing data and compared using the Mann-Whitney-Wilcoxon test.

Estimated charge savings associated with the BPA during the study period were based on charges for thrombophilia tests as listed in the hospital charge master.

\section{RESULTS}

Table 1 summarizes and stratifies BPA adherence. Inpatient alert adherence was significantly higher than outpatient alert adherence $(100 \%$ vs. $16 \%, p<0.001)$. Non-hematology specialists and general medicine providers (internal medicine and family medicine) were more likely to follow the alert than hematology providers $(50 \%$ and $44 \%$ vs. $10 \%, p<0.001$ for both).

When comparing the thrombophilia testing rate before and after the implementation of the BPA, we found that the thrombophilia testing rate did not change significantly in the inpatient setting, but it decreased significantly in the outpatient setting from 0.13 to $0.11(p=0.01)$ post-BPA implementation (Table 2).

During the study period, estimated charges avoided were $\$ 290,430$, based on the assumption that each BPA followed prevented the entire bundle of thrombophilia tests associated with the BPA from being ordered.

\section{DISCUSSION}

An EMR-integrated thrombophilia BPA was effective in influencing thrombophilia test ordering in both inpatient and outpatient settings, particularly among non-hematology specialists and general medicine providers. After hematologists, general medicine providers were the provider group most likely to see the BPA.

In the inpatient setting, the BPA had a remarkable follow rate of $100 \%$, reflecting the fact that most inpatient thrombophilia work-ups are inappropriate. ${ }^{1,2,5}$ Conversely, the BPA was infrequently followed in the outpatient setting and among hematologists. This is unsurprising, as 
Table 1 BPA Outcome by Setting, Provider, and Specialty

\begin{tabular}{llll}
\hline \hline & $\begin{array}{l}\text { Not } \\
\text { followed }\end{array}$ & Followed & $\begin{array}{l}\boldsymbol{p} \\
\text { value }^{*}\end{array}$ \\
\hline Total $(N=185)$ & $\begin{array}{l}122 \\
(66 \%)\end{array}$ & $63(34 \%)$ & - \\
& 122 & $24(16 \%)$ & $<0.001$ \\
$\begin{array}{l}\text { Practice setting } \\
\text { Outpatient }(N=146)\end{array}$ & $\begin{array}{l}(84 \%) \\
\text { Inpatient }(N=39)\end{array}$ & $\begin{array}{l}39 \\
(100 \%)\end{array}$ & \\
$\begin{array}{l}\text { Provider type } \\
\text { MD }(N=119)\end{array}$ & $71(60 \%)$ & $48(40 \%)$ & 0.03 \\
$\begin{array}{l}\text { Non-MD }(N=63) \\
\text { MD role }\end{array}$ & $48(76 \%)$ & $15(24 \%)$ & \\
$\begin{array}{l}\text { Attending physician }(N=77) \\
\text { Resident/fellow }(N=42)\end{array}$ & $57(74 \%)$ & $20(26 \%)$ & $<0.001$ \\
$\begin{array}{l}\text { Provider specialty } \\
\text { Hematologist }(N=73)\end{array}$ & $14(33 \%)$ & $28(67 \%)$ & \\
$\begin{array}{l}\text { Specialist, non-hematology } \\
(N=109)\end{array}$ & $66(90 \%)$ & $7(10 \%)$ & $\begin{array}{l}\text { Ref. } \\
\text { Internal medicine and family }\end{array}$ \\
\begin{tabular}{l} 
medicine $(N=32)$ \\
\hline
\end{tabular} & $18(56 \%)$ & $14(44 \%)$ & $<0.001$ \\
\hline
\end{tabular}

*Fisher's exact test

thrombophilia testing is more often appropriate in the outpatient setting and hematologists are more likely to see complex cases where thrombophilia testing is appropriate. Importantly, the BPA appeared to be highly relevant and actionable for general medicine providers and non-hematology specialists, who followed the alert $40-50 \%$ overall and $20-25 \%$ of the time in the outpatient setting.

Compared to some previously described interventions, EMR-based BPAs without a hard stop on ordering have the benefit of being less labor-intensive for both the stewards of thrombophilia testing and those ordering the tests. ${ }^{3,5}$ EMRbased BPAs have previously been shown to effectively nudge providers towards other forms of guideline-based care, such as for appropriate transfusion thresholds. ${ }^{6}$

Our study reflects the experience of a single institution and is limited by relatively small alert volume and short duration of follow-up. Evaluation of the long-term effects of the BPA and whether it leads to sustainable change will be undertaken.

In conclusion, we found that an EMR-integrated BPA had positive effects on curtailing thrombophilia testing. This is particularly relevant to general medicine providers, who order a substantial proportion of total thrombophilia tests. Similar interventions could be implemented and studied on a larger scale.
Table 2 Thrombophilia Testing Rate Before and After the BPA in Outpatient and Inpatient Settings

\begin{tabular}{llll}
\hline \hline & Pre-BPA $^{\dagger}$ & Post-BPA $^{\dagger}$ & $\boldsymbol{p}$ value $^{*}$ \\
\hline Outpatient & & & \\
PE/DVT cases per month & $\begin{array}{l}285.5 \\
(250-316)\end{array}$ & $\begin{array}{l}281 \\
(272-289)\end{array}$ & 0.91 \\
Tests per month & $\begin{array}{l}36.5 \\
(34-40)\end{array}$ & $\begin{array}{l}31 \\
(20-35)\end{array}$ & 0.03 \\
Testing rate & $\begin{array}{l}0.13 \\
(0.13-0.14)\end{array}$ & $\begin{array}{l}0.11 \\
(0.07-0.12)\end{array}$ & 0.01 \\
Inpatient & $\begin{array}{l}\text { (7) } \\
\text { PE/DVT cases per month }\end{array}$ & $\begin{array}{l}80 \\
(79-88)\end{array}$ & 0.49 \\
Tests per month & $\begin{array}{l}8.5 \\
(6.5-12.5)\end{array}$ & $\begin{array}{l}(4-11) \\
(4-5)\end{array}$ & 0.45 \\
Testing rate & $\begin{array}{l}0.11 \\
(0.09-0.15)\end{array}$ & $\begin{array}{l}0.10 \\
(0.04-0.13)\end{array}$ & 0.30 \\
\hline
\end{tabular}

*Mann-Whitney-Wilcoxon test

† Median (IQR)

Acknowledgements: We gratefully acknowledge Mehran Teymourtash, Melissa Meixsell, and Anibal Perez, RN, for their assistance with data acquisition for this study.

Corresponding Author: Jason Hom, MD; Department of Medicine, Division of Hospital Medicine, Stanford University School of Medicine, 300 Pasteur Drive, HC007, Stanford, CA 94305, USA (e-mail: jasonhom@stanford.edu).

\section{Compliance with Ethical Standards:}

Conflict of Interest: The authors declare that they do not have a conflict of interest.

\section{REFERENCES}

1. Mou E, Kwang $\mathbf{H}$, Hom $\mathbf{J}$, et al. Magnitude of Potentially Inappropriate Thrombophilia Testing in the Inpatient Hospital Setting. J Hosp Med. 2017;12(9):735-738. doi:https://doi.org/10.12788/jhm.2819

2. Cox N, Johnson SA, Vazquez $\mathbf{S}$, et al. Patterns and Appropriateness of Thrombophilia Testing in an Academic Medical Center. J Hosp Med. 2017;12(9):705-709. doi:https://doi.org/10.12788/jhm.2804

3. Shen Y-M, Tsai J, Taiwo E, et al. Analysis of Thrombophilia Test Ordering Practices at an Academic Center: a Proposal for Appropriate Testing to Reduce Harm and Cost. PloS One. 2016;11(5):e0155326. doi:https://doi. org/10.1371/journal.pone.0155326

4. American Society of Hematology | Choosing Wisely. http://www. choosingwisely.org/societies/american-society-of-hematology/. Accessed January 27, 2017.

5. Smith TW, Pi D, Hudoba M, Lee AYY. Reducing inpatient heritable thrombophilia testing using a clinical decision-making tool. J Clin Pathol. 2014;67(4):345-349. doi:https://doi.org/10.1136/jclinpath-2013-201840

6. Goodnough LT, Shieh L, Hadhazy $\mathbf{E}$, Cheng $\mathbf{N}$, Khari $\mathbf{P}$, Maggio $\mathbf{P}$. Improved blood utilization using real-time clinical decision support. Transfusion (Paris). 2014;54(5):1358-1365. doi:https://doi.org/10.1111/ trf. 12445 